Delphine Natali ${ }^{1}$, Georges Cloatre ${ }^{2}$, Christian Brosset ${ }^{3}$, Pierre Verdalle ${ }^{4}$, Alain Fauvy ${ }^{5}$, Jean-Pierre Massart ${ }^{6}$, Quy Vo Van7, Nelly Gerard ${ }^{8}$, Claudia C. Dobler ${ }^{9,10}$, Philippe Hovette ${ }^{11}$

delphinenatali@gmail.com

\title{
Review
}

\section{What pulmonologists need to know about extrapulmonary tuberculosis}

Extrapulmonary tuberculosis (EPT) can affect all organs. Its diagnosis is often challenging, especially when the lung is not involved. Some EPT locations, such as when the central nervous system is involved, are a medical emergency, and some have implications for treatment options and length. This review describes clinical features of EPT, diagnostic tests and treatment regimens.
Cite as: Natali D, Cloatre G, Brosset $C$, et al. What pulmonologists need to know about extrapulmonary tuberculosis. Breathe 2020; 16: 200216.
According to the World Health Organization, tuberculosis disease was the most common cause of death due to an infectious agent in 2019 [1]. The worldwide incidence of tuberculosis is 10 million people per year, with $90 \%$ of cases occurring in 30 high-burden countries. Additionally, around $25 \%$ ( 2.7 billion people) of the world population has latent tuberculosis infection, and is thus at risk of developing tuberculosis. The proportion of extrapulmonary tuberculosis (EPT) is highly variable from one country to another. From 2002 to 2011 in the European Union, EPT without pulmonary involvement represented 19\% of all new tuberculosis cases, ranging from $6 \%$ to 44\%; whereas EPT with pulmonary involvement accounted for $6 \%$. The proportion of new notified tuberculosis cases with pulmonary involvement alone was therefore $75 \%$ [2]. The relative incidence of EPT is increasing: the proportion of new extrapulmonary cases in the European Union increased from $16.4 \%$ of all tuberculosis cases in 2002 to $22.4 \%$ in 2011 [2]. This is probably due to the rise of its risk factors, especially HIV infection [3] and use of anti-tumour necrosis factor $\alpha$ therapies [4, 5].

EPT can potentially affect all organs. Tuberculosis causing pericarditis or involving the central nervous system as well as miliary tuberculosis can present as potentially life-threatening emergencies. Peripheral lymph node and gastrointestinal tuberculosis are common EPT locations, whereas osteoarticular and urogenital tuberculosis can have severe functional consequences.

The diagnosis of EPT, especially in the absence of pulmonary tuberculosis, is often challenging. In $19 \%$ of cases, EPT is not associated with pulmonary tuberculosis [2, 6]. In countries with a low incidence of tuberculosis, physicians may lack awareness of EPT. Non-invasive diagnostic tests often have a lower sensitivity in non-pulmonary specimens, and invasive diagnostic procedures may be needed [7].

In this review, we present the clinical characteristics, diagnostic tools and treatment implications of different EPT manifestations. We focus on EPT presentations with which respiratory professionals are likely less familiar, thus we do not

@ERSpublications

Extrapulmonary tuberculosis can affect any organ, can be potentially life threatening or disabling, poses diagnostic difficulties and may change the type and length of treatment. Looking for concomitant pulmonary tuberculosis is essential. https://bit.ly/2YEaRVb

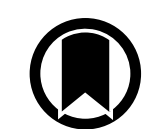

CrossMark

CERS 2021 
elaborate on pleural tuberculosis and mediastinal tuberculous lymphadenopathy in this article.

The information in the article is based on a PubMed search using the keywords "extrapulmonary-tuberculosis", and then the key word of each localisation (e.g. "tuberculous pericarditis"). We also searched for the latest recommendations of professional organisations (e.g. American Thoracic Society, European Respiratory Society).

\section{Diagnosis of EPT}

When EPT is suspected, one should always look for pulmonary tuberculosis. If there is pulmonary involvement, sputum examination for Mycobacterium tuberculosis is a simple, noninvasive test, with good diagnostic yield on direct microscopic examination, molecular testing and cultures. Also, lung involvement might make a patient contagious and has implications for isolation of the patient, protective measures and contact tracing.

Microbiological culture is the gold standard technique to isolate $M$. tuberculosis from a body specimen, permitting also drug-sensibility testing. It takes two (on liquid-based culture media) to 4 weeks (on egg- or agar-based solid-culture media) to grow the organism on culture, but final negative reports are issued only after 8 weeks to allow for a sufficiently long observation period [8]. Acidfast bacilli (AFB) detection on direct microscopic examination (AFB smear) of a body specimen is quick and inexpensive, but has variable sensitivity, requires trained staff, and cannot differentiate M. tuberculosis complex from nontuberculous mycobacteria. Molecular testing can rapidly detect M. tuberculosis complex DNA by PCR, depending on test, in less than $2 \mathrm{~h}$. Some PCR-based tests can also detect mutations associated with drug resistance (amplified M. tuberculosis Direct [9], and GeneXpert MTB/RIF [10]). Sensitivity and specificity of the tests vary, and PCR-based tests do not replace the gold standard cultures. Urine antigen tests for tuberculosis are used in countries with high incidence of HIV and tuberculosis for the diagnosis of miliary tuberculosis in patients with HIV.

The tuberculin skin test and interferon- $\gamma$-release assays are used to diagnose latent tuberculosis infection. Although they can be of some help, they should not be used in the diagnosis of active tuberculosis disease, as a negative result does not rule out tuberculosis with certainty [7].

Depending on the location of tuberculosis in the body, other tests, such as imaging or bodyfluid cytology and chemistry, can be supportive of a tuberculosis diagnosis, but the results of these investigations are not pathognomonic. For example, in lymphocytic pleural, pericardial, peritoneal and joint effusions, an adenosine desaminase (ADA) level $>40 \mathrm{~g} \cdot \mathrm{L}^{-1}$ has a high positive predictive value for the diagnosis of tuberculosis in a patient with a high pre-test probability of tuberculosis, whereas an ADA level $<40 \mathrm{~g} \cdot \mathrm{L}^{-1}$ rules out tuberculosis with high certainty in a patient with a low pre-test probability [7]. In the cerebrospinal fluid (CSF), an ADA level $>10 \mathrm{U} \cdot \mathrm{L}^{-1}$ can be interpreted similarly [7].

Free interferon $-\gamma$ levels in peritoneal fluid have a sensitivity of $93 \%$ and a specificity of $99 \%$; in pleural fluid, their sensitivity is $89 \%$ and their specificity 97\% [7].

An increased erythrocyte sedimentation time (ERS) with a normal C-reactive protein level in the blood is consistent with tuberculosis, but non-specific.

Tissue biopsies showing a caseating granulomatous inflammation are highly indicative of tuberculosis but can also be caused by other infectious organisms. If $M$. tuberculosis cannot be isolated, other diagnoses are not excluded. Sarcoidosis and lymphoma, however, typically present with non-caseating granulomas rather than caseating granulomas like tuberculosis.

\section{Treatment of EPT}

The standard drug therapy for EPT is the same as for pulmonary tuberculosis, that is, rifampicin, isoniazid, ethambutol and pyrazinamide for the first 2 months followed by rifampicin and isoniazid for another 4 months [11]. The treatment regimen needs to be adapted if there is resistance to one or more first-line drugs, but this topic is beyond the scope of our article.

Some locations require intravenous administration of medication, longer duration of treatment, addition of steroids, surgery or emergency empiric treatment [11]. We will elaborate on these issues in the article.

\section{Extrapulmonary thoracic and airways tuberculosis}

Respiratory professionals are usually familiar with tuberculous pleurisy, tuberculous empyema and mediastinal tuberculous lymphadenitis. This article focusses on EPT presentations with which respiratory professionals are likely less familiar, thus we do not elaborate on pleural tuberculosis and mediastinal tuberculous lymphadenopathy in this article.

Respiratory professionals may encounter rare upper airway locations of tuberculosis [12] during bronchoscopy. These lesions of the rhino-pharynx or the larynx can look malignant. Dysphonia is the main symptom of laryngeal tuberculosis, which appears as an ulceroproliferative or infiltrative lesion of the larynx. Tuberculosis of the nasal cavity commonly presents as a chronic unilateral nasal obstruction with purulent discharge, epistaxis and cervical lymph-node enlargement. Odynophagia 
with unilateral ulceration or hypertrophy of a tonsil is the usual feature of oropharyngeal tuberculosis. Rare cases of auricular tuberculosis manifest as chronic purulent otitis media resistant to usual antibiotics, with multiple tympanic perforations. Tuberculous parotitis typically presents as slowly progressive swelling of the parotid gland without constitutional symptoms such as weight loss of fever. The main diagnostic investigation in the above-described locations is a fine needle aspiration biopsy (FNAB) for cytology, AFB smear, cultures and molecular tests [13].

\section{Potentially life-threatening emergencies}

Tuberculous pericarditis, miliary tuberculosis and tuberculosis of the central nervous system are potentially life-threatening and require prompt diagnosis and treatment.

\section{Tuberculous pericarditis}

Tuberculous pericarditis, which affects only $1-2 \%$ of patients with tuberculosis, may lead to (potentially fatal) cardiac tamponade. Rarely, patients develop constrictive pericarditis. Patients with HIV are more likely to develop haemodynamic instability and concurrent myocarditis [14].

\section{Effusive tuberculous pericarditis}

Effusive tuberculous pericarditis presents as subacute pericarditis, with constitutional symptoms such as weight loss and fever usually preceding the onset of dyspnoea on exertion and chest pain. On chest radiography, the cardiac silhouette is flask-shaped and enlarged (figure 1), and signs of pulmonary, pleural or mediastinal tuberculosis may be present.

Echocardiography shows a thickened pericardium containing various amounts of hypoechoic fluid with fibrinous strands (figure 2) [15] and detects cardiac tamponade, if present. A computed tomography of the chest is the best imaging modality to detect pleuro-pulmonary and mediastinal involvement, whereas magnetic resonance imaging (MRI) of the heart can detect a pericardial abscess.

Emergency pericardiocentesis should be performed for cardiac tamponade. Pericardial fluid is usually bloodstained, lymphocytic and exudative with an ADA level above $40 \mathrm{~g} \cdot \mathrm{L}^{-1}[7,16]$. AFB smear and culture have a low sensitivity: $0-42 \%$ and 50-65\%, respectively [7]. Data regarding molecular testing in pericardial fluid are limited. Pericardial biopsy has a sensitivity of $73-100 \%$ [7]. For the treatment of effusive tuberculous pericarditis that does not require an emergency pericardiocentesis, inferior pericardiotomy with drain placement should be considered to avoid cardiac tamponade. It also
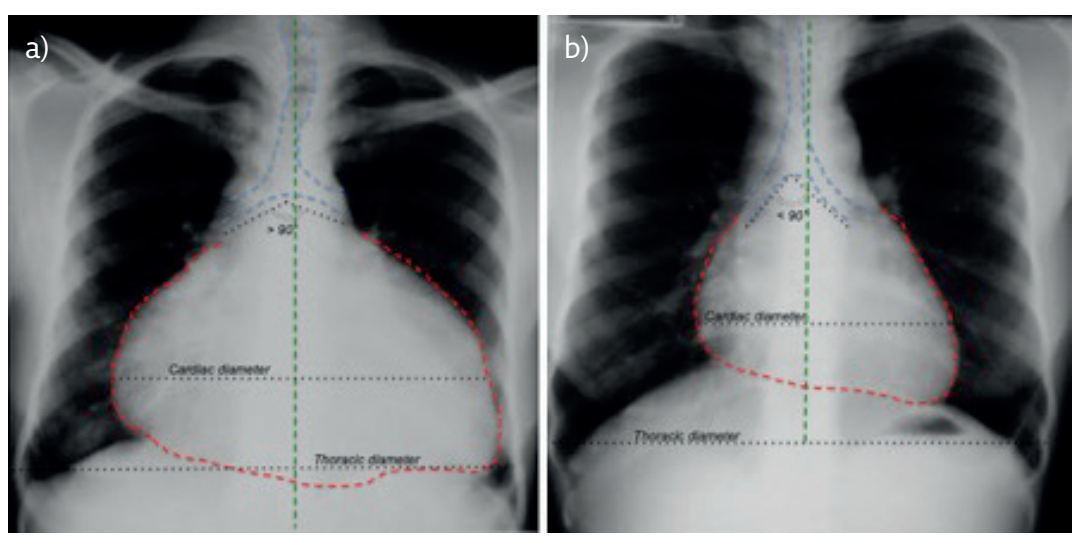

Figure 1 Tuberculous pericarditis: chest radiograph. a) Severe pericardial effusion. The heart silhouette (red) is enlarged (cardio-thoracic ratio $>50 \%$ ), with symmetric expansion compared to the spine (green): "flask-shaped" or "water-bottle" or "chicken on a fence" sign. The angle between the right and left main bronchi (blue) is $>90^{\circ}$ : "widening of the carinal angle" sign. b) same patient, after pericardiocentesis. Figure modified and reproduced with permission from Pierre L'Her (HIA Percy, Clamart, France).

allows direct visualisation of the pericardium (resembling millet seeds), and pericardial fluid and biopsy sampling. Main differential diagnoses of effusive tuberculous pericarditis are pericarditis caused by other infectious organisms, malignancy and haemopericardium.

Particularity of treatment of tuberculous effusive pericarditis: addition of corticosteroids (dosage: $1 \mathrm{mg} \cdot \mathrm{kg}^{-1}$ per day) should not be used routinely but selectively in patients at high risk of progression to constrictive pericarditis $[11,17]$. At presentation, urgent pericardiotomy may be required to obtain diagnostic samples and to avoid tamponade.

\section{Constrictive pericarditis}

Constrictive pericarditis is rare and typically presents as progressive dyspnoea on exertion with signs of right heart failure [18].

Chest radiography may show a ring of calcification around the heart. Echocardiography demonstrates increased pericardial thickness and signs of constriction with abnormal septal motion, respiratory variation of ventricular filing, bi-atrial enlargement and dilated inferior vena cava and hepatic veins [19]. Right-heart catheterisation allows hemodynamic evaluation of constrictive pericarditis, with increased right atrial pressure and a dip and plateau pattern in the right
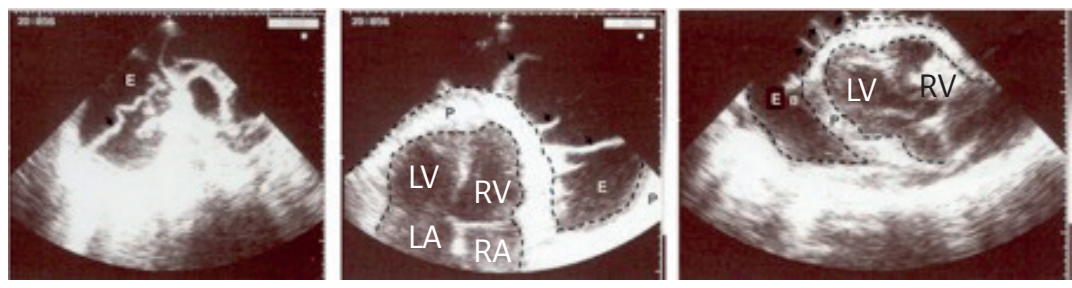

Figure 2 Tuberculous pericarditis: echocardiography. Echocardiography showing a typical tuberculous effusive pericarditis with effusion $(E)$, pericardial thickness $(P)$, and perpendicular pericardial strands (black arrows). $R V$ : right ventricle, $L V$ : left ventricle, $R A$ : right atrium, $L A$; left atrium. Figure modified and reproduced with permission from Georges Cloatre. 
ventricular pressure tracing [20]. Cardiac computed tomography and $\mathrm{MRI}$ provide detailed information about the extent of pericardial thickening and calcification [21].

Main differential diagnoses are a post-radiation therapy complication, connective tissue diseases, constrictive pericarditis due to sarcoidosis, malignancy and other infective causes.

Pericardiectomy, often necessary to control heart failure, permits pericardial biopsy for diagnostic purposes.

\section{Miliary tuberculosis}

Miliary tuberculosis is due to lymphohaematogenous spread of M. tuberculosis, during primary infection or reactivation. It is a lethal form of disseminated tuberculosis (i.e. infection which involves the blood stream, bone marrow, liver or two or more non-contiguous body sites) [22, 23].

Clinical manifestations can be subacute with fever, dysfunction of one or more organs and night sweats or fulminant with multiorgan failure, septic shock and acute respiratory distress syndrome. The lungs have a miliary aspect (like millet seeds), and most commonly the lymphatic system, bones, joints, liver, central nervous system and adrenal glands are involved as well [24].

Chest radiography shows bilateral uniformly distribution of miliary nodules, measuring 1-3 mm in diameter. This aspect is best seen on a computed tomography scan of the chest. Correlated with the clinical presentation, these imaging findings are highly suggestive of miliary tuberculosis. Miliary tuberculosis is often associated with haematologic anomalies, disseminated intravascular coagulation, elevated ERS, hyponatraemia (due to syndrome of inappropriate antidiuretic hormone secretion $(\mathrm{SIADH})$ ), hypercalcaemia and aseptic pyuria [24]. The chest radiograph and computed tomography chest demonstrate numerous bilateral disseminated micronodules (figure 3) [24]. The
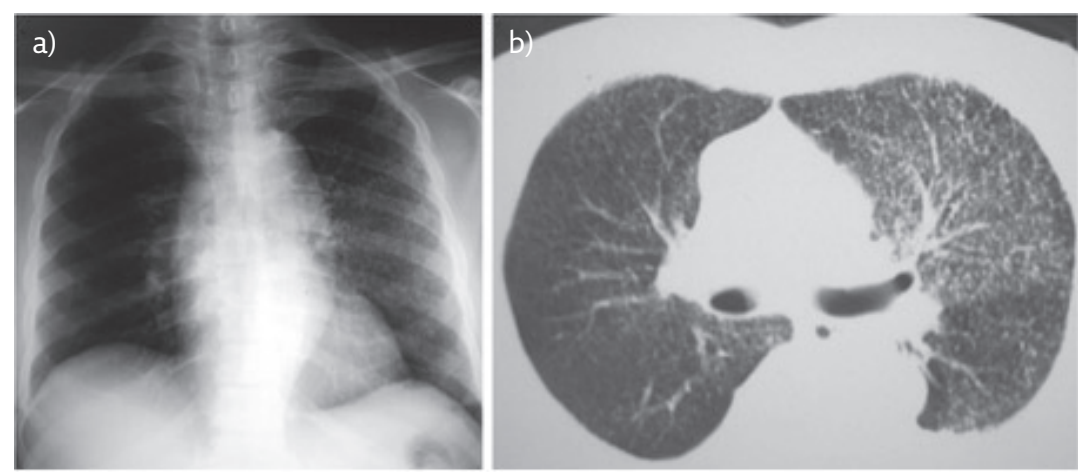

Figure 3 Miliary tuberculosis. a) Chest radiograph and b) computed tomograph of the chest of a 40-year-old African man, HIV negative, presenting with subacute onset of high fever $\left(40^{\circ} \mathrm{C}\right)$, shortness of breath and general malaise. Imaging studies showed bilateral micronodular lung infiltrates, resembling millet seeds. In this case the lesions were predominantly on the left side. Sputum acid-fast bacilli (AFB) smears were negative. Diagnosis was obtained by positive AFB smear from bronchial washings sampled during bronchoscopy. Figure modified and reproduced with permission from Pierre L'Her (HIA Percy, Clamart, France). finding of choroidal tubercles (Bouchut's tubercles) on fundoscopy is pathognomonic for miliary tuberculosis [24].

Sampling of involved organs for AFB smear, culture, molecular testing and cytology/histology generally allows the diagnosis [24]. In patients with HIV, an inexpensive urine antigen test (lateral-flow urine lipoarabinomannan assay: detection of the mycobacterial cell wall glycolipid lipoarabinomannan) has a good sensitivity, especially if CD4 cells are $<100 \mathrm{~mm}^{-3}$, and the results can be obtained in $<1 \mathrm{~h}$ [25-27].

Miliary tuberculosis is a medical emergency and empiric treatment should be initiated promptly. Treatment duration and regimen should be adapted to the involved organs.

\section{Tuberculous meningitis and encephalitis}

The onset of tuberculous meningitis is often subacute [28], with fever, stiffness, vomiting, altered consciousness, and cranial nerve palsies (especially cranial nerves VI, III, IV and VII [29, 30]). At an advanced stage, patients suffer from paralysis, stupor, coma and seizures. Complications, such as hydrocephalus, hyponatraemia and vision loss, are common. If the disease is left untreated, death occurs within 5-8 weeks.

A computed tomography scan or MRI of the brain can demonstrate thick basilar exudates, cerebral infarcts, hydrocephalus and optochiasmatic arachnoiditis [31]. The combination of hydrocephalus, basal enhancement and infarction has a specificity of $95-100 \%$ for the diagnosis of tuberculous meningitis [29].

CSF examination shows a high opening pressure $\left(25 \mathrm{cmH}_{2} \mathrm{O}\right)$ in $50 \%$ of cases [29], lymphocytic pleiocytosis, elevated protein concentration and a ratio of CSF to plasma glucose $<0.5$ in $95 \%$ of cases [32]. ADA levels over $8 \mathrm{UI} \cdot \mathrm{L}^{-1}$ support a diagnosis of tuberculous meningitis [7, 33]. AFB smears are positive in only $10-30 \%$ of patients but remain positive days after initiating antituberculous treatment [7]. The diagnostics yield increases with CSF volume (up to $10-15 \mathrm{~mL}$ ) and number (up to four) of CSF samples. Sensitivity of CSF culture is 45-70\% [7]. PCR (detecting DNA of M. tuberculosis only) has a sensitivity of $62 \%$ and a specificity of 98\% [7, 34], whereas GeneXpert (real-time PCR detecting both DNA of M. tuberculosis and mutation of $r p o B$ gene, which indicates a resistance to rifampicin) has a sensitivity of 59-66\% [35, 36].

Differential diagnoses of tuberculous meningitis are fungal, bacterial or viral meningitis, neurobrucellosis, neurosyphilis and neoplastic meningitis.

As soon as tuberculous meningitis is suspected, empiric antituberculous treatment should be initiated without delay [11]. However, the diagnosis of meningeal tuberculosis can be challenging, as M. tuberculosis is often not found in the CSF. In the 
absence of a diagnostic reference standard with high accuracy, empiric treatment with antituberculous medication for tuberculous meningitis and with conventional antibiotics for pyogenic meningitis is a reasonable approach in cases where the cause of meningitis is unclear. After an initial 2-month phase of isoniazid, rifampicin, pyrazinamide and ethambutol, an additional 7-10 months second phase of isoniazid and rifampicin is recommended [11]. Corticosteroid therapy reduces the risk of death and disabling neurological deficit amongst HIVnegative patients with tuberculous meningitis [37]. A paradoxical worsening of tuberculous meningitis on antituberculous treatment can occur, and can be treated with corticosteroids.

\section{Brain tuberculomas}

Patients with brain tuberculomas are often asymptomatic or may have seizures [38]. Brain tuberculomas can be associated with tuberculous meningitis [39].

Computed tomography with contrast and MRI of the brain show a solitary or multiple enhancing ring lesions with surrounding oedema, not distinguishable from cerebral ring enhancing lesions due to causes other than tuberculosis [31].

If no other organs are affected by tuberculosis, diagnosis of brain tuberculomas is challenging. They should be suspected in patients with one or more mass lesions of the brain and risk factors for tuberculosis. Lumbar puncture should be performed with caution in patients with large tuberculomas, because of the risk of brainstem herniation. Needle biopsy of the brain lesion for AFB smear, culture, molecular testing and pathology is indicated, if there is no option to obtain specimens from other involved organs $[38,40]$. In settings with a low incidence of tuberculosis, empiric treatment for toxoplasmosis should be considered in patients with HIV before performing a needle biopsy.

The main differential diagnoses of brain tuberculomas are neurocysticercosis, cryptococcoma, toxoplasmosis, brain abscess, lymphoma and primary or secondary tumours of the brain.

Information about risk factors for either tuberculosis or malignancy (e.g. immunosuppression, history of malignancy) are important to inform the diagnosis.

For brain tuberculomas, the treatment protocol is the same as for tuberculous meningitis. Glucocorticoids are used in cases of severe brain oedema with mass effect, intracranial hypertension and concomitant meningitis. A paradoxical reaction on antituberculous treatment may occur and brain tuberculomas may persist despite adequate antibiotic treatment.

\section{Tuberculous spinal arachnoiditis}

Tuberculous spinal arachnoiditis affects the spinal cord, meninges and nerve roots at any level, in varying combinations. It is the consequence of breakdown of granulomas within these structures, which then become progressively encased in tuberculous exudates, forming a fibrous mass. The main risk factor for spinal arachnoiditis is tuberculosis spondylitis (Pott's disease), especially if the spondylitis is long-standing and initiation of antituberculous treatment is delayed. The clinical manifestations are various, ranging from symptoms caused by upper and/or lower motoneuron involvement, to radiculomyelitis with paraparesis, radicular pain and bladder dysfunction.

Spinal MRI shows nodular arachnoiditis. In the CSF, protein levels are typically high with or without pleiocytosis. AFB smear, culture and molecular testing of CSF facilitate the diagnosis of tuberculosis in cases with associated meningitis. In the absence of meningitis or tuberculosis in other organs, biopsy of the spinal lesion is necessary to make a definite diagnosis [38].

The main differential diagnoses of tuberculous spinal arachnoiditis are cytomegalovirus infection and syphilis.

There is no strong evidence for management of spinal arachnoiditis. Experts propose an extension of treatment up to 12 months, and to consider use of corticosteroids [41]. Surgery is indicated in selected patients with poor response to adequate medical treatment.

\section{Frequent locations of EPT}

The most frequent locations of EPT, after tuberculous pleurisy, are tuberculous lymphadenitis, osteoarticular tuberculosis, uro-genital tuberculosis and tuberculosis of the gastrointestinal tract.

\section{Tuberculous lymphadenitis}

In non-HIV patients, tuberculous peripheral lymphadenitis often presents as a swelling of a single group of lymph nodes at a single site, mostly in the cervical, submandibular or supraclavicular areas, progressing over 1-2 months, without constitutional symptoms [42]. On physical examination, the lymph nodes are painless, firm, fixed and the overlying skin may be thickened or red. Complications such as ulceration, fistula or abscess formation may be seen (figure 4). Concomitant active pulmonary involvement is rare [43]. In contrast, patients with HIV frequently have symptoms such as fever, night sweats and weight loss, there is often more than one lymph node site involved and concomitant pulmonary tuberculosis is common.

Ultrasonography is a simple, non-invasive, inexpensive and widely available imaging technique to better characterise peripheral lymph nodes (figure 5) [44]. A computed tomography scan (figure 5) or MRI of the neck can help to characterise the lymph nodes, but are usually not necessary. 

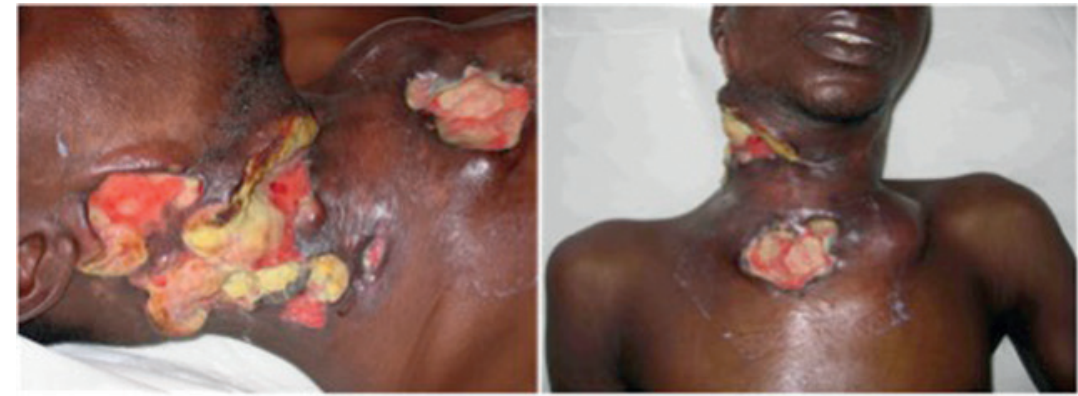

Figure 4 Tuberculous peripheral lymphadenitis clinical presentation. Cervical tuberculous lymphadenitis complicated by scrofuloderma in a non-HIV patient (Ivory Coast). Figure modified and reproduced with permission from Pierre L'Her (HIA Percy, Clamart, France) and Philippe Hovette.

Material from peripheral lymph nodes can be easily, safely and inexpensively obtained by ultrasound-guided FNAB. However, the AFB smear has a low sensitivity (20\%), and culture, with a sensitivity of $60 \%$, takes time. Additional molecular tests increase the diagnostic yield of the smear: PCR has a sensitivity of 20-50\%, and GeneXpert of $26-73 \%$ [45-47]. A typical pathology finding on FNAB of caseating granulomatous inflammation will usually suffice to start empiric treatment for tuberculosis. Excisional biopsy for pathology, AFB smear and culture, and molecular testing, has globally a higher sensitivity (80\%) and may be considered if the findings from a FNAB are inconclusive or if a differential diagnosis is more probable.

Histiocytic necrotising lymphadenitis (Kikuchi disease), malignancy and lymphoma, nontuberculous mycobacterial infections, cat scratch disease and bacterial lymphadenitis are the most frequent differential diagnoses that can be ruled out by lymph node sampling.

Tuberculous lymphadenopathy can also involve mediastinal or abdominal lymph nodes, either by lymphatic or haematogenous spread. Abdominal lymph nodes have the same imaging features as peripheral lymph nodes. Symptoms depend on the lymph node location. Hepatic lymph nodes can leads to jaundice, portal vein thrombosis and portal hypertension. Compression of the renal artery by tuberculous lymphadenopathy can cause renovascular hypertension. Intestinal obstruction may occur in the case of mesenteric lymph nodes (figure 6). FNAB or excisional biopsy for cytology or pathology, AFB smear and culture, and molecular testing, has the same advantages and limitations as in peripheral lymph nodes. Lymph node needle aspiration by endobronchial ultrasound (EBUSTBNA) and/or oesophageal ultrasound (EUS-FNA) are the techniques of choice in the diagnostic work-up of both mediastinal and abdominal lymph nodes [48, 49].

In peripheral tuberculous lymphadenitis, a paradoxical reaction may occur during treatment, due to an immune response to dying $M$. tuberculosis. It manifests either with an increased size of preexisting enlarged lymph nodes and/or appearance of new lymph nodes and/or spontaneous discharge. Observation may be sufficient, but aspiration, surgical excision, nonsteroidal anti-inflammatory drugs or corticosteroids may be required to improve the patient's symptoms. New investigation of the lymph node is not necessary if a microbiological diagnosis of tuberculosis has been made and drug sensitivities are known. Other cases have to be assessed on a case-by-case basis.

\section{Tuberculous spondylitis or Pott's disease}

Clinicians should be aware of this disease manifestation, because it can be associated with
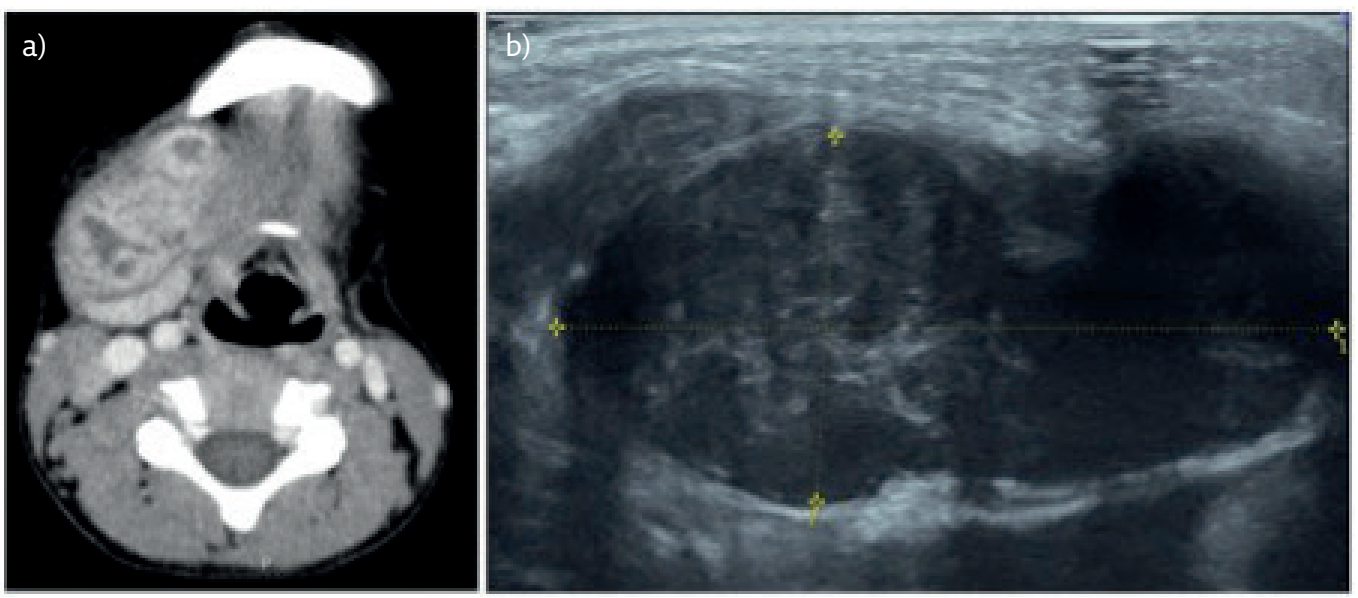

Figure 5 Tuberculous peripheral lymphadenitis imaging. Computed tomography scan and ultrasound of an 8-year-old HIV-negative Vietnamese boy presenting with a 1-month history of a right cervical mass, which was initially misdiagnosed as a complication of a tooth abscess. He had no concomitant pulmonary tuberculosis. An acid-fast bacilli (AFB) smear of the fine needle aspiration biopsy of the lymph node was positive. a) Cervical computed tomography scan. The tuberculous lymph node is enlarged with central caseating areas of low-density. b) Cervical ultrasound. The tuberculous lymph node is hypoechogenic, with thin layers, matting and surrounding soft tissue oedema. Figure modified and reproduced with permission from Pierre Verdalle and Do Van Tu (Radiology Dept, Hanoi French Hospital, Hanoi, Vietnam). 

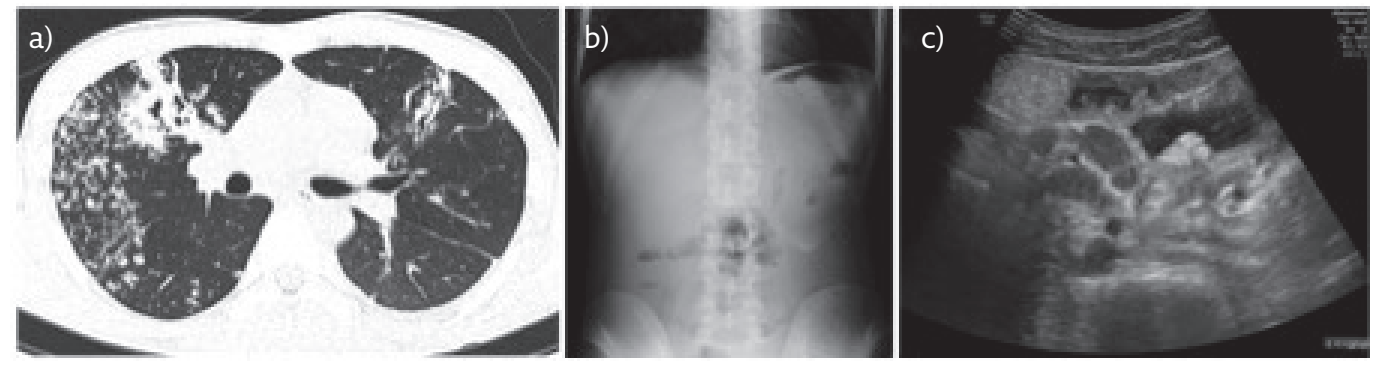

Figure 6 Tuberculous mesenteric lymphadenitis: paradoxical reaction. A 23-year-old Vietnamese man with a past medical history of closure of interatrial septal communications, HIV negative, presented with a weight loss of $30 \mathrm{~kg}$ weight loss in 1 year. a) The computed tomography scan of the chest showed changes typical for pulmonary tuberculosis. A sputum acid-fast bacilli (AFB) smear was positive. 10 days after starting anti-tuberculous treatment, he presented to the emergency department with severe epigastric pain without passing stool or gas for 48 h. b) An abdominal radiograph showed a periumbilical hydro-aeric level suggestive of bowel obstruction. c) An ultrasound of the abdomen revealed enlarged mesenteric lymph nodes compressing the bowel. The patient made a full recovery with hydratation, naso-gastric aspiration and anti-tuberculous treatment. Figure modified and reproduced with permission from Delphine Natali and Nguyen The Hung (Radiology Dept, Hanoi French Hospital, Hanoi, Vietnam).

severe complications (figures 7-9). The infection is commonly located in the lower thoracic and upper lumbar region. It starts in the intervertebral joints, spreads to the adjacent vertebral body and then to the adjacent intervertebral disc space, leading to vertebral collapse and gibbus deformity, and risk of medullar compression with resulting paraplegia or tetraplegia. Formation of cold abscesses around the sites of infection is common, and these abscesses may drain in a fistula to the skin. Bone lesions occurring at more than one level are a characteristic feature of tuberculous spondylitis.

A chronic and progressively increasing (over weeks to months) localised pain is often the presenting symptom [50-52]. Patients walk with head and chest thrown backward, protuberant abdomen and legs wide apart, taking short steps to avoid jarring of the spine (Alderman's gait). Constitutional symptoms and lung involvement are rare.
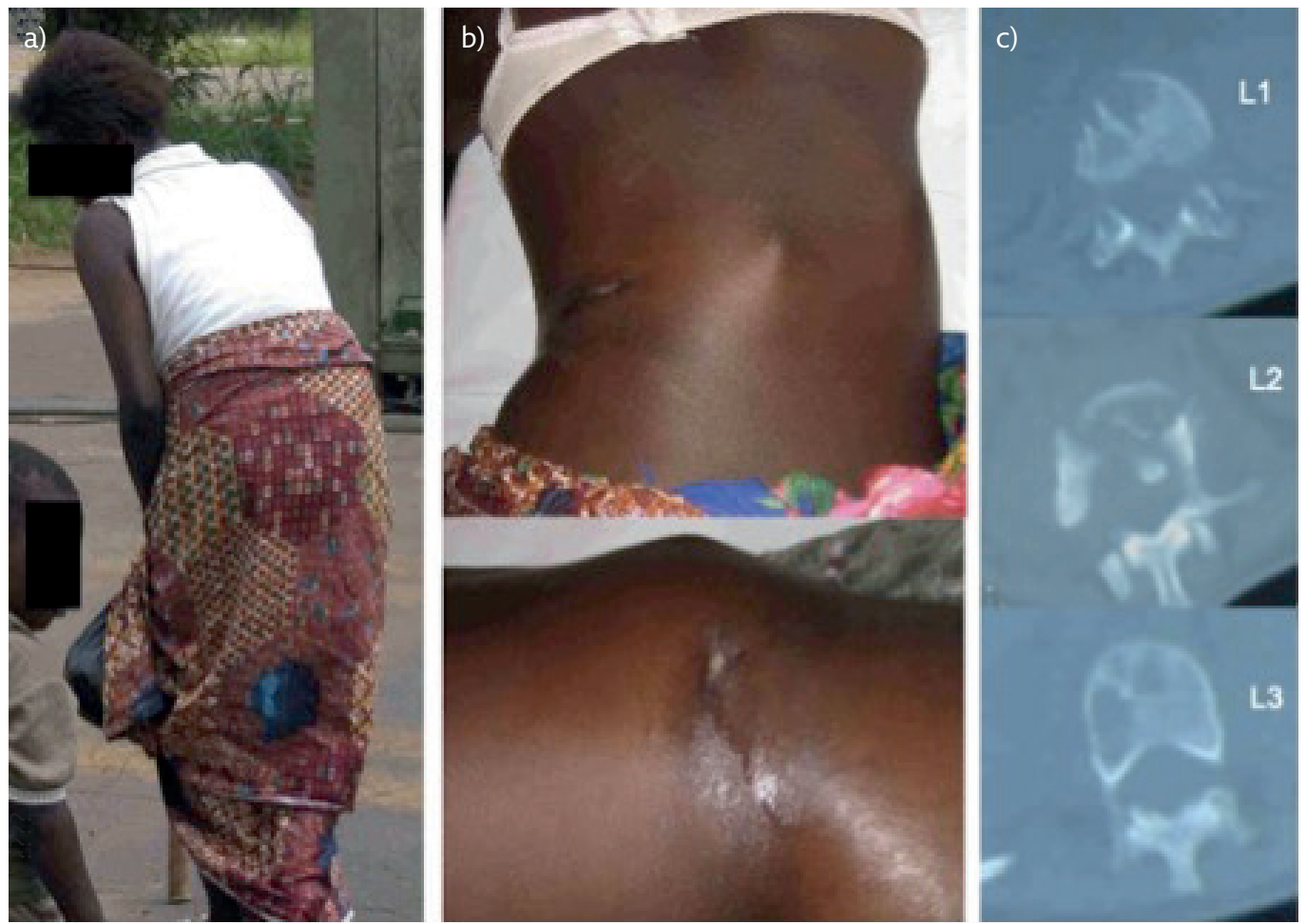

Figure 7 Tuberculosis spondylitis (Pott's disease) at the upper lumbar level. This 29-year-old Ivorian woman was misdiagnosed with lumbago for 2 years. She had no fever and no constitutional symptoms. She was HIV-negative. A computed tomography-guided fine needle aspiration biopsy revealed a diagnosis of tuberculosis. a) Lumbar spine deformity. b) Draining sinuses with fluid discharge; lumbar spine angulation. c) Lumbar spine computed tomography scan showing osteolytic lesions and destruction of the vertebrae bodies on lumbar (L) level (L1, L2, L3). Figure modified and reproduced with permission from Philippe Hovette. 

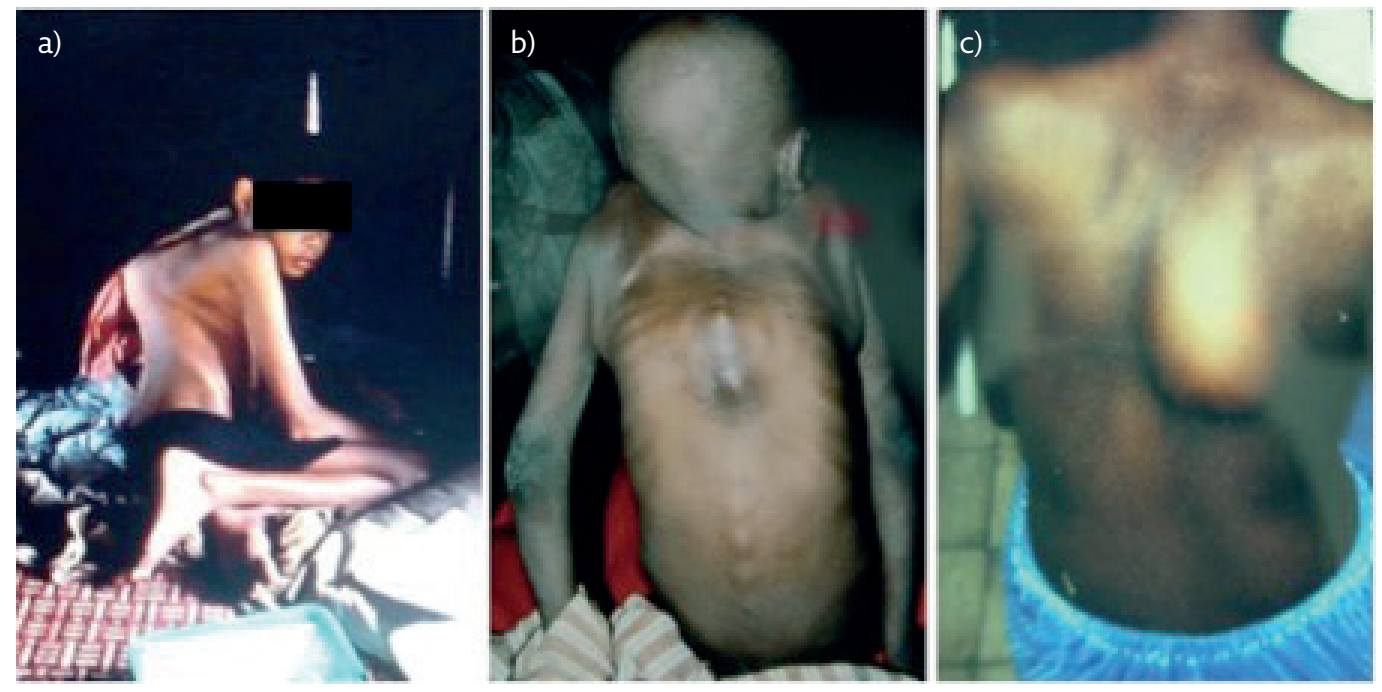

Figure 8 Tuberculosis spondylitis (Pott's disease) at the thoracic level. Typical gibbus deformation with kyphosis: a) in a Cambodian boy and b) in an Ivorian boy. c) Paravertebral abscess formation in a Cambodian boy. Figure modified and reproduced with permission from Pierre L'Her (HIA Percy, Clamart, France) and Philippe Hovette.

A spinal radiograph typically shows demineralisation of the vertebrae, paravertebral abscesses and obliteration of disc spaces with anterior wedging and angulation. A computed tomography scan of the spine can show destruction of vertebral bodies, loss of disk height, erosion of end-plates, subchondral cysts and paravertebral abscesses [53]. Spinal MRI is the investigation of choice to evaluate soft tissue extension of the infection, compression of the spinal cord or associated spinal arachnoiditis. [54]. Intervertebral disk infection leads to vertebral osteomyelitis with destruction of two or more adjacent vertebrae and apposed end-plates. Vertebra involvement leads to vertebral osteonecrosis, sub-ligamentous abscesses and vertebral collapse. Adjacent soft-tissue dissemination causes paravertebral or iliopsoas abscess formation.

In the absence of concomitant pulmonary tuberculosis, the diagnosis of tuberculous spondylitis is often challenging. A computed tomography scan-guided FNAB allows sampling for AFB smear, culture, molecular testing and pathology. GeneXpert testing on tissue samples may improve the diagnostic yield [55, 56]. Culture of the fluid discharged from draining sinuses may be useful; however, this material is often colonised with pyogenic bacteria or fungi.

Differential diagnoses are numerous: spondylarthropathy, vertebral body collapse due to osteopenia, spondylitis caused by other pathogens, pyogenic spinal infection and malignancy.

In tuberculous spondylitis, the length of treatment may be prolonged to 9-12 months [11]. Surgery is needed in cases with severe neurologic deficits, or if neurologic symptoms worsen during appropriate treatment, or if the patient has a kyphosis $>40^{\circ}$ at the time of presentation. If tuberculous arthritis occurs in a prosthetic joint, removal of the artificial joint is required.
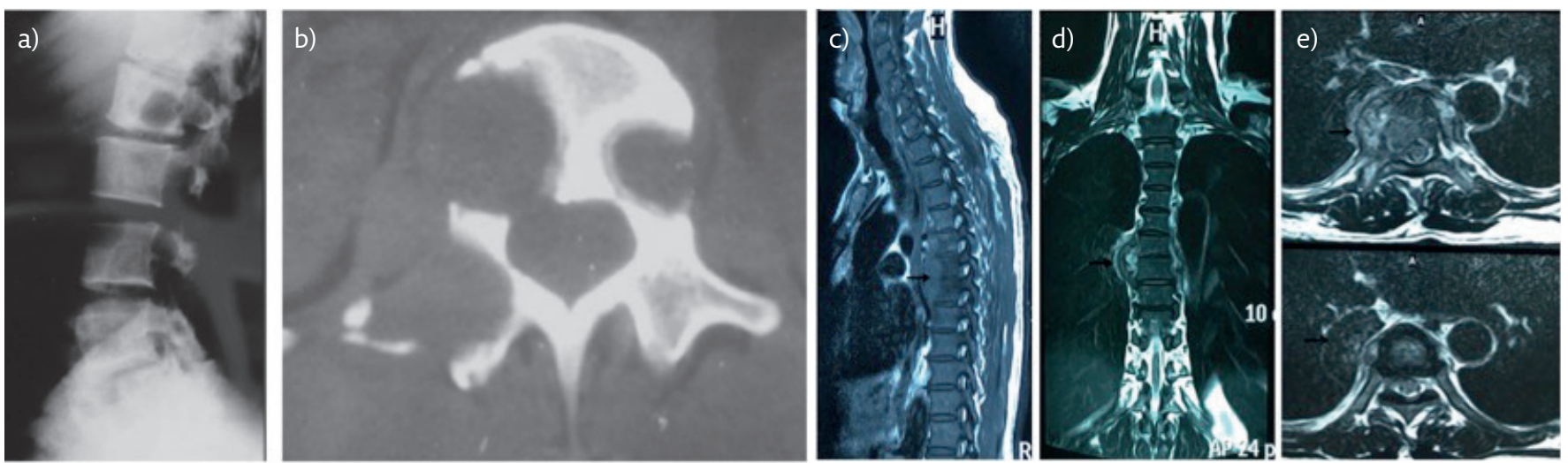

Figure 9 Tuberculosis spondylitis (Pott's disease) imaging. a) Thoracic spine radiograph. Osteolytic lesions of the vertebral bodies and loss of disk height. b) Corresponding computed tomography scan of the thoracic spine. c-e) Magnetic resonance imaging of the spine. Tuberculosis spondylitis with paravertebral abscess (arrow) at the D5-D6 level. Figure modified and reproduced with permission from Pierre L'Her, and Prof. Philippe Hovette and Christian Brosset. 

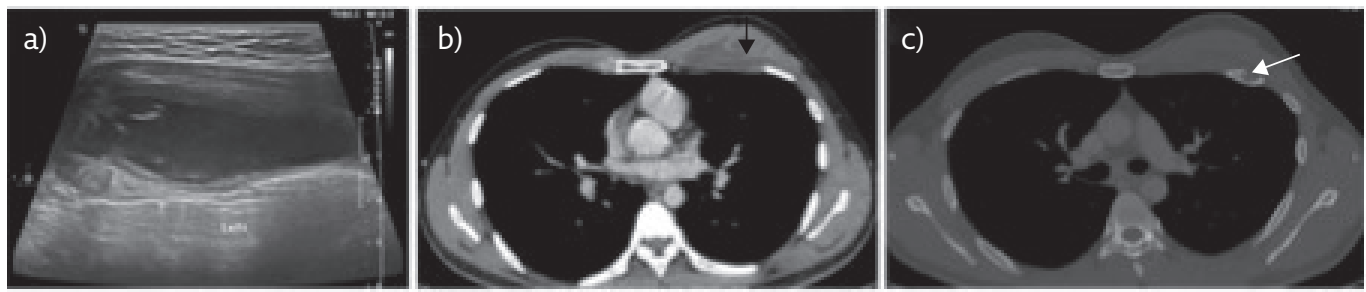

Figure 10 Cold abscess of the chest wall. Left third rib cold abscess in a 28-year-old Vietnamese man, HIV-negative, presenting with a painless left thoracic chest wall mass for 1 month. a) Thoracic ultrasound showed a hypoechogenic heterogenous lesion. b) Computed tomography of the chest confirmed this lesion, black arrow) with c) third rib involvement, white arrow). Surgical exploration of the lesion revealed yellow thick fluid with presence of Mycobacterium tuberculosis. Figure modified and reproduced with permission from Do Van Tu and Nguyen The Hung Hung (Radiology Dept, Hanoi French Hospital, Hanoi, Vietnam).

\section{Tuberculous osteomyelitis}

Tuberculous osteomyelitis can affect any bone. It develops slowly over time, usually at a single site. The clinical presentation depends on the location, for example, a cold abscess of the chest wall can be present due to tuberculous osteomyelitis of a rib (figure 10) or dactylitis can be present due to tuberculosis of the bones of the fingers or toes.

A radiograph of the involved bone shows cystic changes; computed tomography scan and MRI help to determine the lesions' extension.

Diagnosis is obtained by FNAB, with specimens processed for AFB smear, culture, molecular tests and pathology.

\section{Tuberculous arthritis}

Tuberculosis can affect joints in two ways: it can cause infection of a native or prosthetic joint (tuberculous arthritis) or an immune-mediated joint inflammation (Poncet's disease).

Tuberculous arthritis (figure 11) typically develops in a single location, either hip or knee and presents as a subacute painful swelling with loss of joint function. On physical examination, the joint appears cold, with sometimes draining sinuses to the skin or joint effusion. The most advanced cases may have joint deformities. Constitutional symptoms are rare. A radiograph of the joint shows local soft tissue swelling and calcifications, osteoporosis, bone erosion and joint collapse [57]. A computed tomography or MRI of the affected joint will help to determine the extent and severity of the lesions. Synovial fluid analysis has a low diagnostic yield, whereas synovial biopsy for AFB smear, culture, molecular testing and pathology has a very good diagnostic yield [7]. Culture of the fluid coming from draining sinuses can potentially facilitate a diagnosis of tuberculosis; however these samples are often colonised with pyogenic bacteria and fungi. Differential diagnoses are degenerative and bacterial or fungal arthritis.

Poncet's Disease is an immune-mediated acute symmetric oligo- or polyarthritis involving large and small joints, associated with tuberculosis [58]. There is no tuberculosis infection in the joints, so samples from other organs (if applicable) are required to establish a diagnosis. The prognosis with antituberculous treatment is good, avoiding any joint destruction.

\section{Peritoneal tuberculosis}

The usual clinical presentation of peritoneal tuberculosis is a slow accumulation of ascites fluid over weeks or months, sometimes associated with abdominal pain and fever [59].

Exploratory paracentesis is the first diagnostic step [60]. The fluid is straw coloured, lymphocytic, with a serum-ascites albumin gradient below 1 and elevated ADA levels [7]. AFB smear, cultures and GeneXpert have a low sensitivity, <5\%, 45-69\% and $18 \%$ respectively [7]. Peritoneal biopsy by laparoscopy (for AFB smear, culture, molecular and pathology tests) may be required to establish the diagnosis [61]. The peritoneum has the appearance of millet seeds.

Differential diagnoses are end-stage liver disease, bacterial or fungal peritonitis and malignancy.

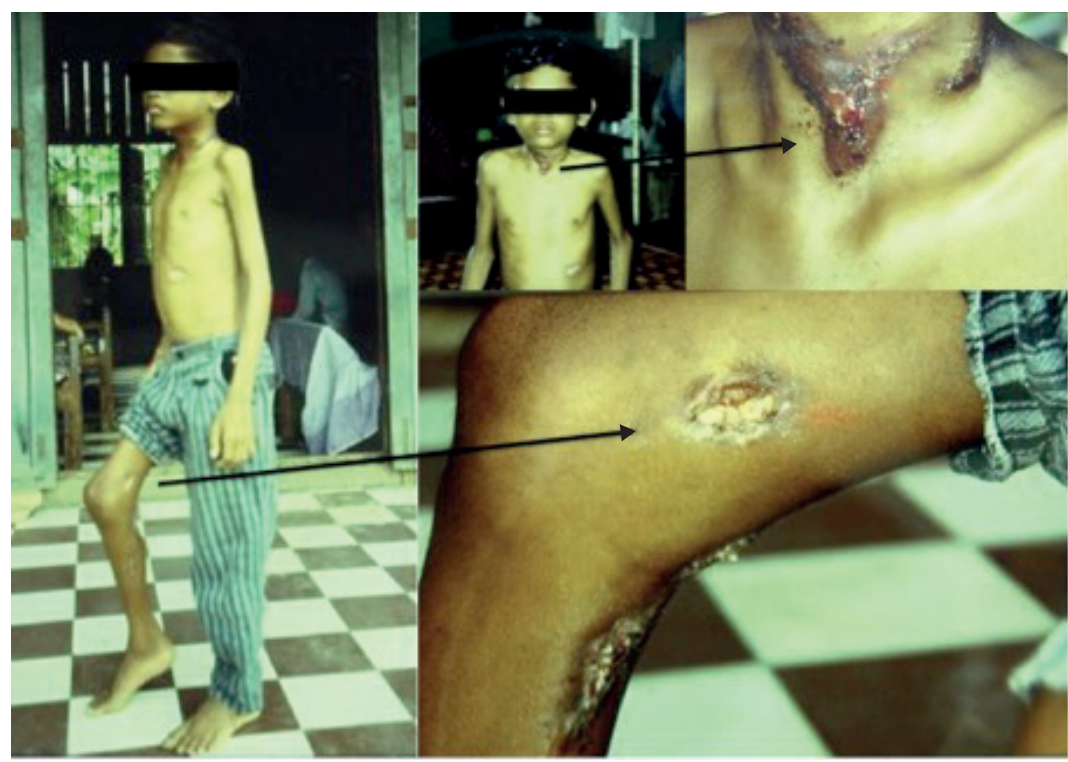

Figure 11 Tuberculous arthritis of the knee. A 14-year-old Cambodian boy limping because of tuberculous arthritis of the right knee with scrofuloderma and fistulisation to the skin. Physical examination revealed also cervical lymphadenopathy with scrofuloderma, and a chest radiograph (not shown here) demonstrated bilateral active pulmonary tuberculosis. Figure modified and reproduced with permission from Pierre L'Her (HIA Percy, Clamart, France). 

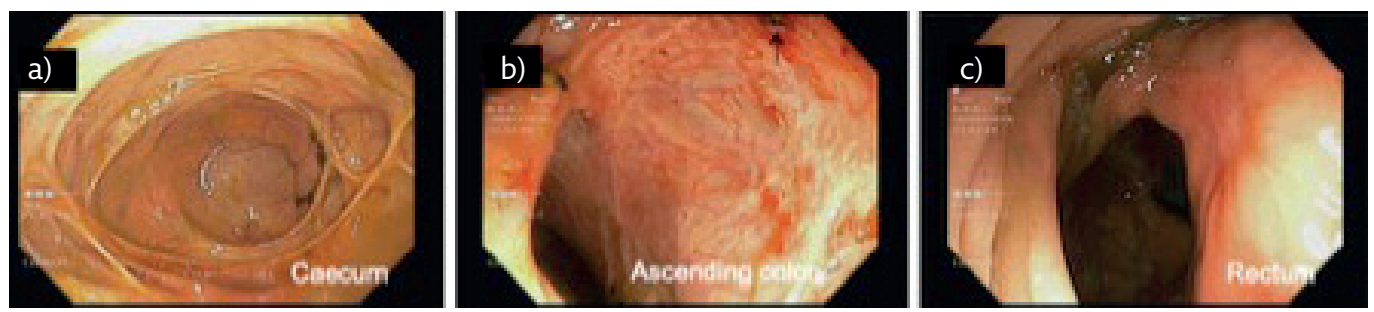

Figure 12 Gastrointestinal tuberculosis colonoscopy findings. A 40-year-old Korean woman, HIV-negative, underwent colonoscopy during a systematic health-check for a work permit. a) The caecum had multiple circular strictures. There were b) inflammatory and superficial ulcerations in the right colic angle and c) a scar of past ulceration in the rectum. There was no concomitant pulmonary tuberculosis. Biopsy of the ascending colon ulceration showed caseating granulomatous inflammation, with acid-fast bacilli (AFB) smear and culture negative but GeneXpert positive without rifampicin resistance. Figure modified and reproduced with permission from Annie Lion (Gastro-enterology, CH Fougères, Fougères, France).

\section{Hepatic tuberculosis}

Hepatic tuberculosis is more often observed in miliary tuberculosis than in an isolated local disease form. In the latter case, the patient often presents with abdominal pain, constitutional symptoms and jaundice due to compression of the biliary tract by enlarged lymph nodes. The main challenge is to start potentially hepatotoxic antituberculous treatment despite hepatic dysfunction (elevated transaminases). If hepatic tuberculosis is the main cause for elevated transaminases, improvement of the liver function on antituberculous treatment is to be expected.

Ultrasound, computed tomography scan and MRI show hepatic nodules with calcifications and peripheral enhancement and hepatic lymph nodes $[62,63]$.

If the liver is the only disease location, a diagnosis is obtained by a liver biopsy for AFB smear (sensitivity $25 \%$ ), PCR (sensitivity $86 \%$ ) and pathology [64, 65].

Main differential diagnoses are liver cancer or metastasis and liver abscess.

\section{Tuberculosis of the gastrointestinal tract}

Tuberculosis can affect the entire digestive tract, but the most frequent location is the ileocaecal region. The patient usually presents with abdominal distension, diarrhoea, nausea, vomiting, gastrointestinal bleeding, ascites and constitutional symptoms [66].

Imaging studies (computed tomography scan and ultrasound) may reveal wall thickening in the ileocaecal region and lymphadenopathies with central low density.

Colonoscopy (figure 12) shows ulcers, nodules, a deformed ileocaecal valve, strictures, pseudopolyps and fibrous bands, and allows biopsy sampling. AFB smear and culture of intestinal biopsy have a low sensitivity; thus, molecular testing and pathology facilitate the diagnosis.

The most important differential diagnosis is Crohn's disease [67], which typically shows noncaseating granulomas on intestinal biopsies.

Intravenous treatment is sometimes required in patients with severe vomiting or patients with bowel obstruction due to abdominal tuberculosis. Surgery may be needed in complications, such as gastrointestinal perforation, abscess, fistula, bleeding, or severe obstruction. Intravenous tuberculosis treatment with all first-line drugs can be challenging because of difficulties procuring the intravenous formulations and/or difficulties of administering intravenous medications in an ambulatory care setting.

\section{Renal and urological tuberculosis}

Tuberculosis involving the urinary tract (kidneys, ureters, bladder and urethra) commonly presents as aseptic pyuria and/or a microscopic haematuria with a low pH [68]. Frequent urination, dysuria and low back pain are common, whereas systemic symptoms are rare. In advanced stages, renal insufficiency and high blood pressure can occur. Patients with HIV are prone to kidney or prostate abscesses. Computed tomography scans of the abdomen and pelvis show concomitant upper and lower urinary tract involvement with strictures throughout the urinary tract, asymmetric caliectasis, calcifications, hydronephrosis, bladder contraction and renal damage [69]. Urine AFB smear and culture have a sensitivity of $14-39 \%$ and 45-70\%, respectively [7]. Renal or bladder biopsies have a sensitivity of $86-94 \%$ [7]. Differential diagnoses are malignancy and Mycobacterium bovis cystitis occurring after intravesical Bacillus Calmette-Guérin instillation for treatment of bladder cancer.

Less commonly, tuberculosis can lead to renal parenchymal lesions. Glomerulonephritis can occur due to tuberculosis infection of the kidney. Interstitial nephritis can be due to development of tuberculous granuloma or to an immunologic reaction to rifampicin [70]. Amyloidosis can also develop during tuberculosis infection, due to chronic inflammation, which triggers glomerular deposition of amyloid substance.

\section{Male genital tuberculosis}

The entire male genital tract (prostate, seminal vesicles, vas deferens, epididymis, testicles, Cooper 
glands and penis) can be affected by tuberculosis. Chronic orchi-epididymitis is the most frequent form of male genital tuberculosis, typically presenting with a scrotal nodule, epididymal hardening, a scrotal fistula, a hydrocele or male infertility. Penile tuberculosis is very rare and presents as a genital ulcer. The diagnosis is often challenging, relying on biopsy of the involved location, in the absence of concomitant pulmonary or urological tuberculosis.

\section{Female genital tuberculosis}

Female genital tuberculosis is a common cause of infertility in countries with a high incidence of tuberculosis. It generally spares the myometrium and presents as a chronic salpingitis or endometritis. Other clinical manifestations include chronic pelvic or abdominal pain and menstrual disorders.

A hysterosalpingram shows fallopian tube obstruction or constriction and/or uterine cavity adhesion or deformity. A diagnosis is obtained via biopsy of the involved structures or culture of menstrual fluid.

In genitourinary tuberculosis, surgery may be needed on a case-by-case basis, including for example stenting or percutaneous nephrostomy, surgical nephrectomy, dilatation or reconstruction of strictures.

\section{Other forms of EPT}

\section{Adrenal gland insufficiency}

Tuberculosis can lead to adrenal gland insufficiency (Addison disease) through gradual bilateral adrenal destruction after haematogenous spread from an infection elsewhere in the body.

Early in the disease, abdominal computed tomography or MRI show enlarged adrenal glands. Later on, caseous nodules and fibrosis replace the adrenal gland tissue. Thus, after 2 years, the adrenal glands appear normal or smaller, with or without calcifications [71, 72].

Clinical signs of chronic adrenal insufficiency are malaise, fatigue, weakness, anorexia, weight loss, hypotension, electrolytes abnormalities (hyponatraemia, hyperkalaemia) and hyperpigmentation. Acute adrenal insufficiency with shock can occur during severe infection or an acute major stressful event.

A low cortisol level at 08:00 $\mathrm{h}$ and after adrenocorticotropic hormone (ACTH) stimulation demonstrates adrenal gland insufficiency.

Supplementation with hydrocortisone is recommended in adrenal gland insufficiency. Recovery of adrenal gland function occurs in only $50 \%$ of cases after antituberculous treatment and long-term corticosteroid replacement may be required.
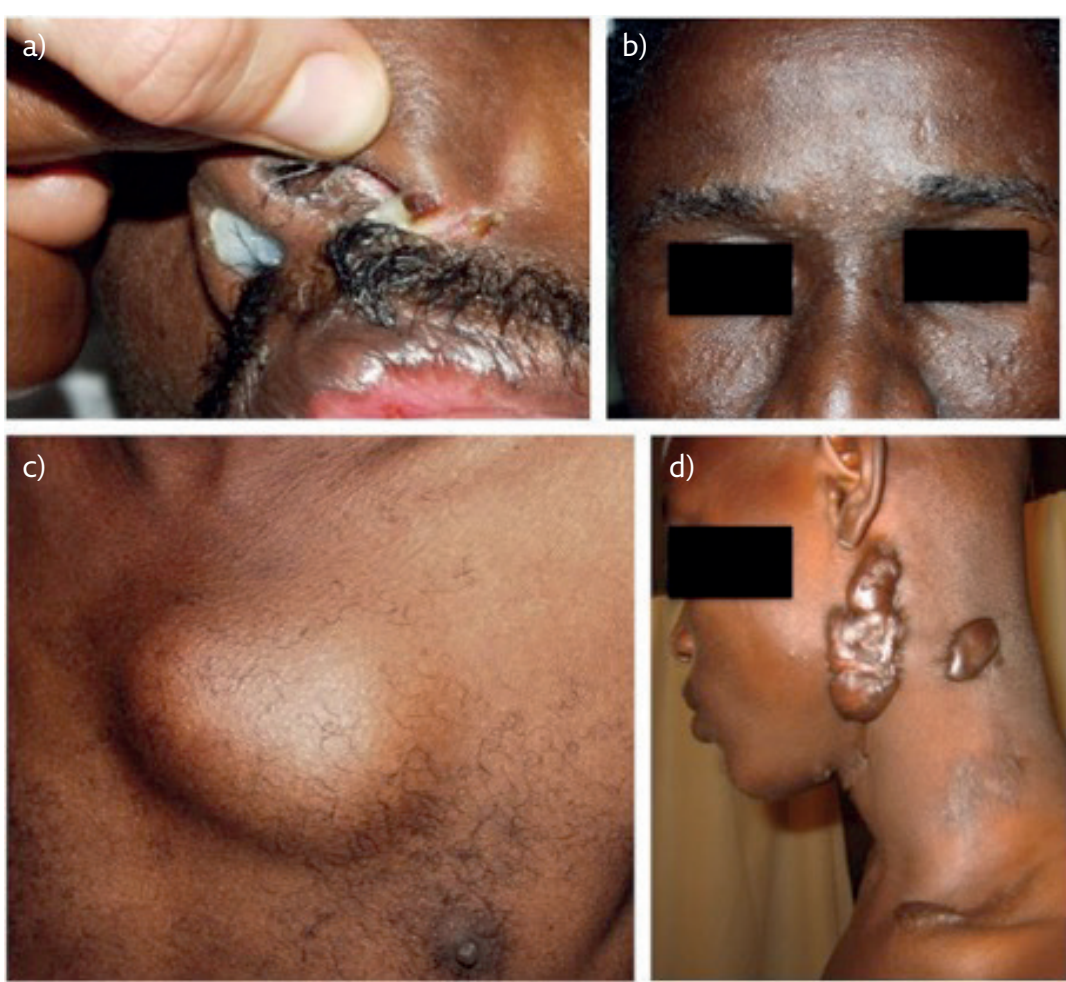

Figure 13 Skin tuberculosis. a) Tuberculosis cutis orificialis. b) Tuberculids: papular lesions of the forehead. c) Tuberculous cold skin abscess. d) Keloid scar of a previous scrofuloderma. Figure modified and reproduced with permission from Fabrice Simon (Infectious and Tropical Diseases, Val De Grâce, Paris, France) and Philippe Hovette.

\section{Cutaneous tuberculosis}

Cutaneous tuberculosis (figure 13) is uncommon and none of the lesions is specific [73]. As presented in table 1 , it can be classified based on the route of infection (system 1) or the bacterial load (system 2). Clinical manifestations are numerous and presented in table 2 . The diagnostic tools depend on the type of lesion.

Table 1 Classification of cutaneous tuberculosis

\begin{tabular}{ll}
\hline Classification system 1 & Classification system 2 \\
\hline Exogenous & Multibacillary \\
- Tuberculous chancre & - Tuberculosis chancre \\
- Tuberculosis verrucosa cutis & - Scrofuloderma \\
Endogenous & - Tuberculosis cutis orificialis \\
Contiguous & - Acute miliary tuberculosis \\
- Scrofuloderma & - Gummatous tuberculosis \\
- Tuberculosis cutis orificialis & \\
Haematogenous & Paucibacillary \\
- Acute miliary tuberculosis & - Tuberculosis verrucosa cutis \\
- Metastatic tuberculosis abscess & - Lupus vulgaris \\
- (gummatous tuberculosis) & Non-bacillary hypersensitivity \\
- Papulonecrotic tuberculid & reaction (tuberculids): \\
Lymphatic & - Papulo necrotic tuberculid \\
- Lupus vulgaris & - Lichen scrofulosorum \\
\hline
\end{tabular}


Table 2 Clinical aspects of cutaneous tuberculosis

\begin{tabular}{|c|c|}
\hline Primary inoculation tuberculosis & $\begin{array}{l}\text { - Occurs in non-sensitised children } \\
\text { - After minor traumatism } \\
\text { - Red-brown papules, faces and extremities }\end{array}$ \\
\hline Tuberculosis verrucosa cutis & $\begin{array}{l}\text { - Occurs after direct inoculation of a previously sensitised host } \\
\text { - Lilaceous or brownish-red warty growth } \\
\text { - Knees, elbows, hands, feet, and buttocks }\end{array}$ \\
\hline Lupus vulgaris & $\begin{array}{l}\text { - Chronic and progressive } \\
\text { - Clinical findings are variable } \\
\text { - Lesions persist for years. }\end{array}$ \\
\hline Scrofuloderma & $\begin{array}{l}\text { - Firm, painless nodules and may ulcerate } \\
\text { - Extension from underlying bone, joints, or lymph nodes. }\end{array}$ \\
\hline Miliary tuberculosis & $\begin{array}{l}\text { - Immunocompromised patients } \\
\text { - Small red papules, ulcers, and abscesses } \\
\text { - Poor prognosis }\end{array}$ \\
\hline Tuberculid & $\begin{array}{l}\text { - Generalised exanthema in patients with good immunity to tuberculosis } \\
\text { - Erythema induratum of Bazin: recurring nodules or lumps on the back of the legs } \\
\text { - Papulonecrotic tuberculid crops of recurrent crusted skin papules on knees, elbows, buttocks } \\
\text { - Lichen scrofulosorum: eruption of small follicular papules in young adults with underlying } \\
\text { tuberculosis. }\end{array}$ \\
\hline
\end{tabular}

Indeed, for tuberculid lesions, which are due to cutaneous immunological reaction triggered by $M$. tuberculosis infection elsewhere in the

\section{Self-evaluation questions}

1 Tuberculous pericardial effusion:

a) Is an emergency because of the risk of cardiac tamponade.

b) Needs confirmation by right heart catheterisation.

c) May have a typical aspect of thickened pericardium with fibrinous strands on echocardiography.

d) May require addition of corticosteroids to antituberculous treatment to avoid progression to constrictive pericarditis.

2 Tuberculous meningitis:

a) Usually presents as fulminant meningitis with high-grade fever.

b) Can be associated with Bouchut's tubercles on funduscopic examination.

c) Is a contra-indication to corticosteroid therapy.

d) Is a potentially life-threatening emergency.

3 Tuberculous lymphadenitis:

a) Can present with scrofuloderma.

b) Always needs pathological confirmation by excisional biopsy.

c) Is sometimes complicated by paradoxical lymph node enlargement during treatment.

d) Is highly contagious.

4 Tuberculous spondylitis (Pott's disease):

a) Is often revealed by a progressively increasing localised pain.

b) Can induce gibbus deformity.

c) Needs prolonged antituberculous treatment.

d) Can be complicated by an ilio-psoas abscess. body, the clinician should look for another site of tuberculosis to assess the diagnosis of tuberculosis. In contrast, skin biopsy for pathology, AFB smear and culture are useful for the diagnosis of cutaneous tuberculous lesions containing bacilli.

\section{Ocular tuberculosis}

Ocular tuberculosis may occur due to a direct invasion by $M$. tuberculosis. The most common presentation of ocular tuberculosis is uveitis. The patient typically complains of progressive blurry vision, light sensitivity, red eye or eye floaters. Eye examination most often shows posterior uveitis, but anterior, intermediary and pan-uveitis are possible.

The diagnosis is challenging in the absence of other organs involved, and is based on iris or retinochoroidal biopsy with $\mathrm{PCR}$.

Bouchut's tubercles are pathognomonic of miliary tuberculosis and can be seen during a funduscopic examination.

\section{Conclusion}

EPT has a large spectrum of clinical presentations, poses diagnostic challenges and sometimes requires adaptation of treatment. Table 3 summarises clinical characteristics, diagnostic approaches and treatment of different forms of EPT. 


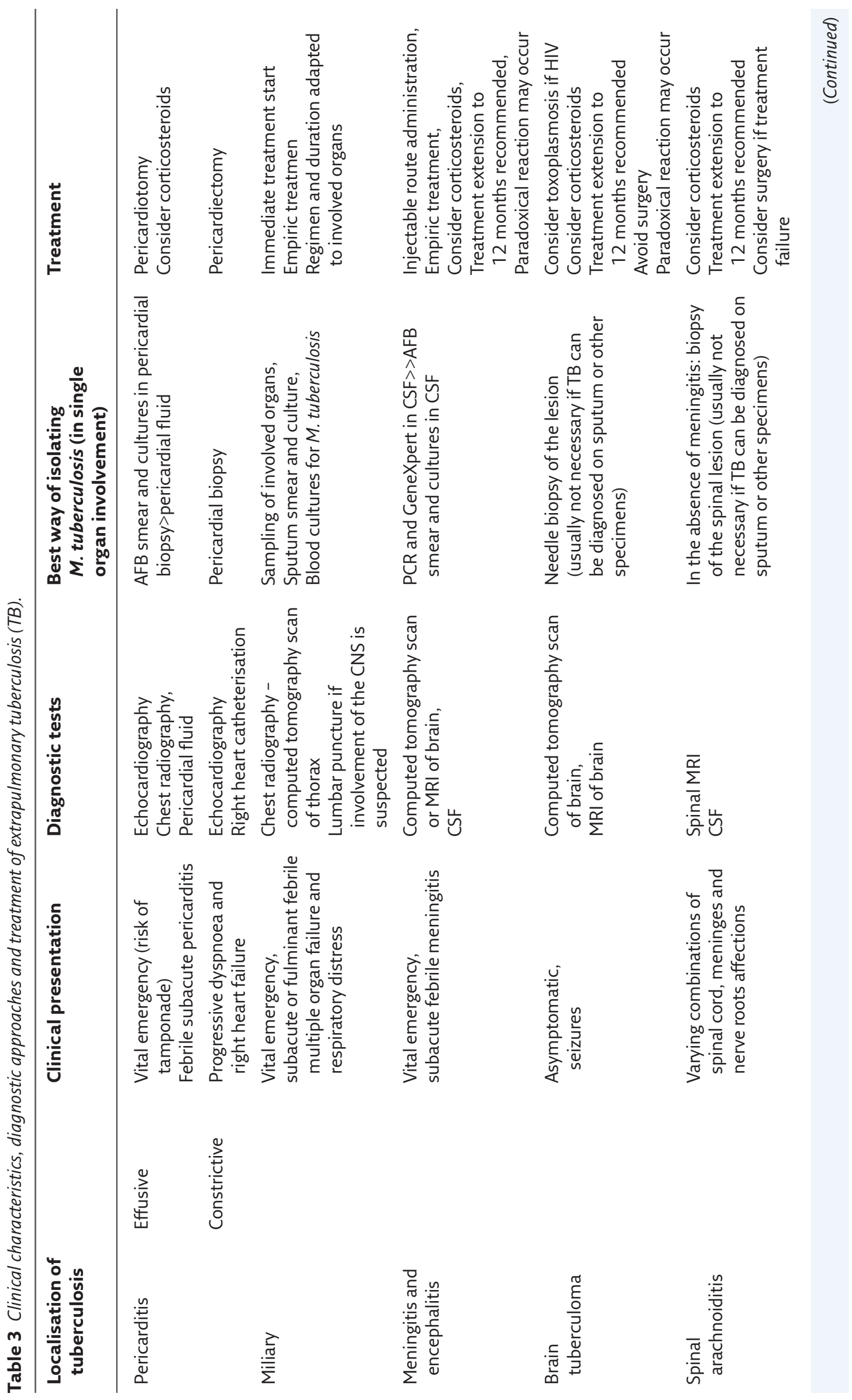




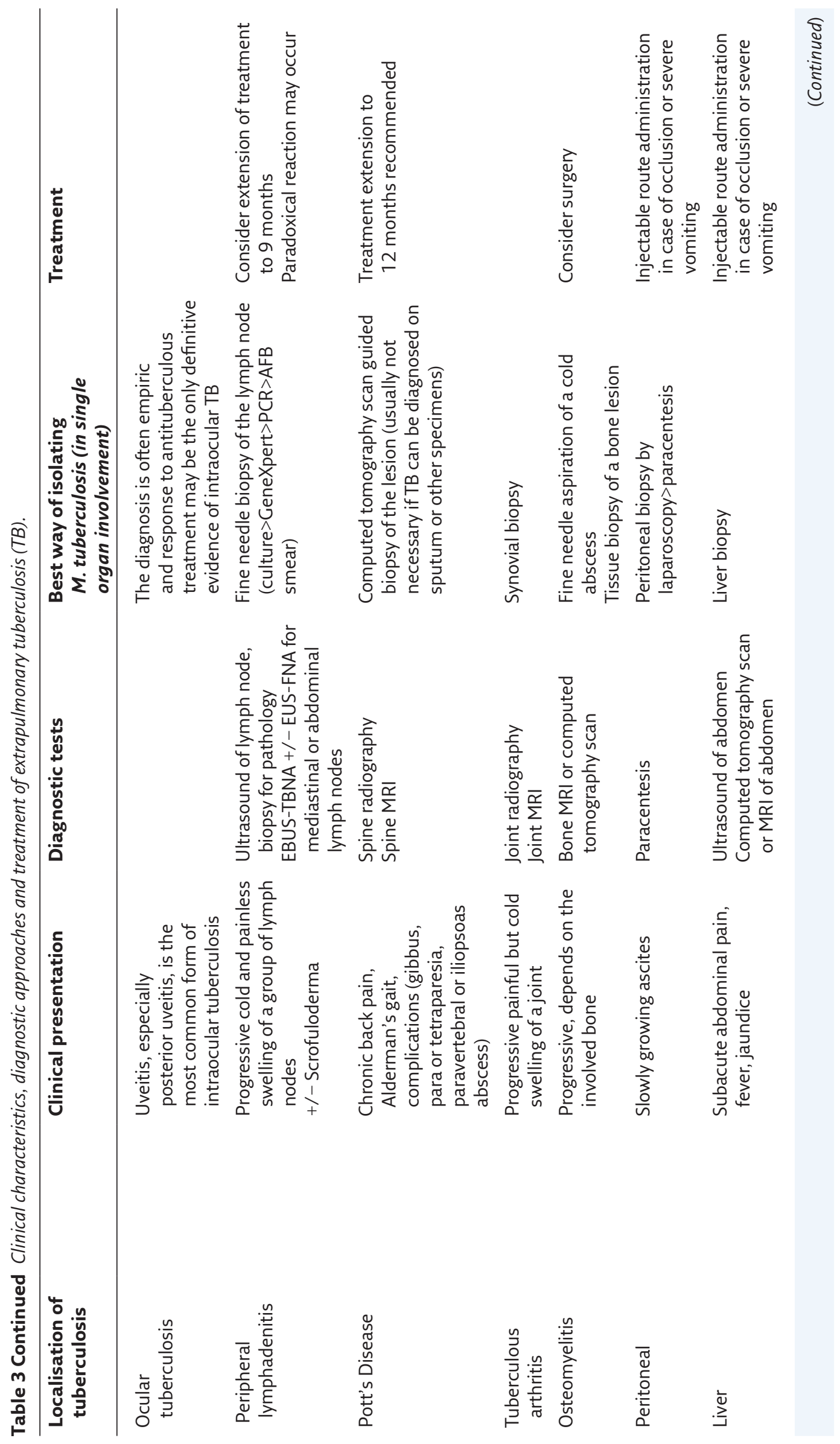




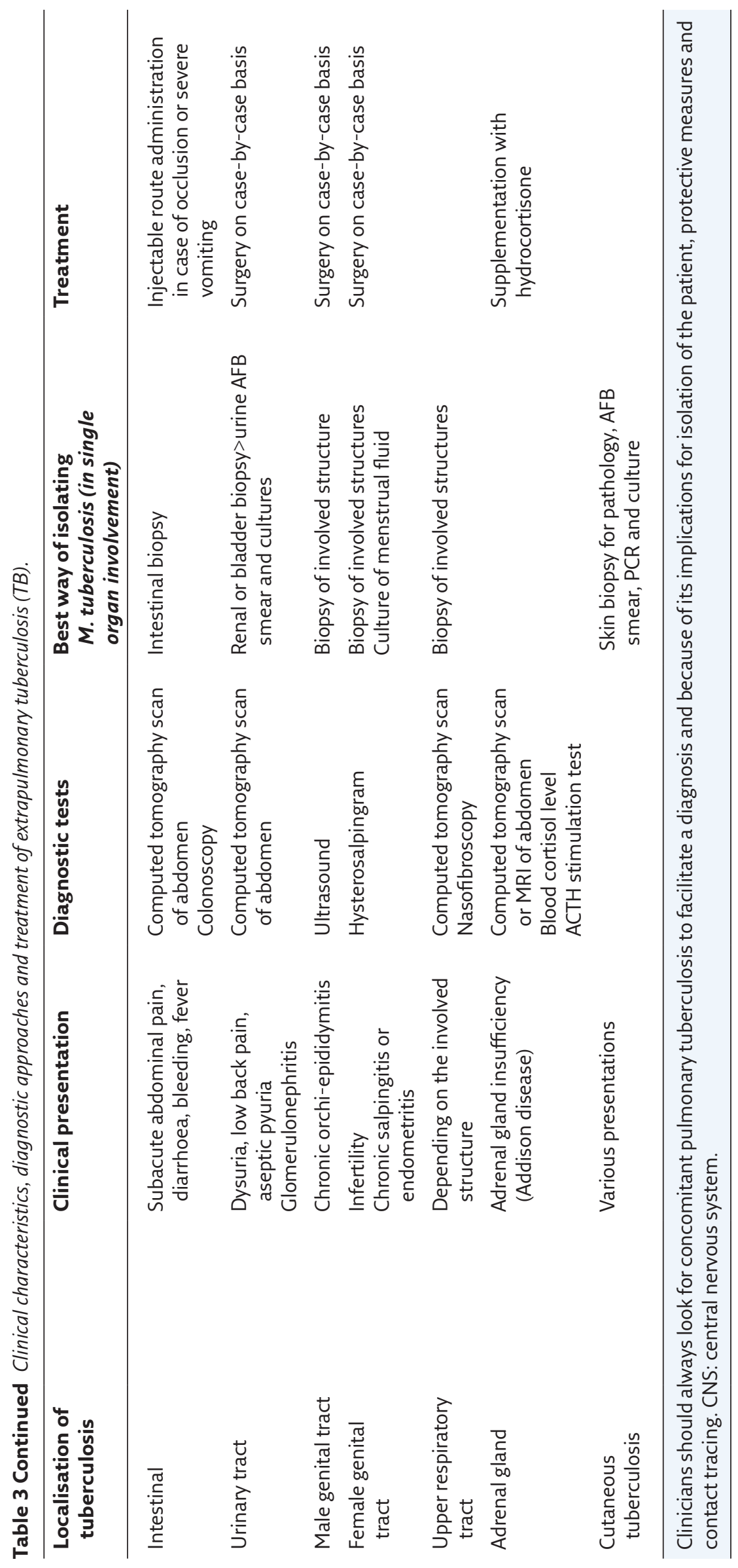




\section{Key points}

- Extrapulmonary tuberculosis (EPT) can affect any organ and can be potentially life threatening or disabling.

- Looking for concomitant pulmonary tuberculosis is essential to facilitate a diagnosis and because of its implications for isolation of the patient, protective measures and contact tracing.

- Diagnosis of EPT is often challenging and is based on identification of M. tuberculosis on specimens from involved organs by direct AFB smear, culture and molecular tests, combined with pathology findings and clinical presentation.

- $\quad$ Presence of EPT may change the type and length of treatment.

Affiliations

Delphine Natali ${ }^{1}$, Georges Cloatre ${ }^{2}$, Christian Brosset ${ }^{3}$, Pierre Verdalle ${ }^{4}$, Alain Fauvy ${ }^{5}$, Jean-Pierre Massart ${ }^{6}$, Quy Vo Van ${ }^{7}$, Nelly Gerard ${ }^{8}$, Claudia C. Dobler9,10, Philippe Hovette ${ }^{11}$

${ }^{1}$ Respiratory Medicine, Hanoi French Hospital, Hanoi, Vietnam. ${ }^{2}$ Cardiology, Hanoi French Hospital, Hanoi, Vietnam. ${ }^{3}$ Neurology, Hanoi French Hospital, Hanoi, Vietnam. ${ }^{4}$ Ear-Nose-Throat Diseases, Hanoi French Hospital, Hanoi, Vietnam. ${ }^{5}$ Orthopedic Surgery, Hanoi French Hospital, Hanoi, Vietnam. ${ }^{6}$ Gastro-Enterology, Hanoi French Hospital, Hanoi, Vietnam. ${ }^{7}$ Urology, Hanoi French Hospital, Hanoi, Vietnam. ${ }^{8}$ Dermatology, Hanoi French Hospital, Hanoi, Vietnam. ${ }^{9}$ Dept of Respiratory Medicine, Liverpool Hospital, Sydney, Australia. ${ }^{10}$ The George Institute for Global Health, University of New South Wales, Sydney, Australia. ${ }^{11}$ Internal Medicine, Hanoi French Hospital, Hanoi, Vietnam.

\section{Acknowledgement}

Pr Pierre L'Her, Respiratory Medicine, HIA Percy, Clamart, France; Dr Annie Lion, Gastro-enterology, CH Fougères, Fougères, France; Pr Fabrice Simon, Infectious and Tropical Diseases, Val De Grâce, Paris, France: for sharing photographs and clinical cases. Dr Do Van Thu and Dr Nguyen The Hung, Radiology Department, Hanoi French Hospital, Hanoi, Vietnam: for support with imaging.

Conflict of interest

None declared.

\section{References}

1. World Health Organization Global Tuberculosis Report. https://apps.who.int/iris/bitstream/handle/10665/3360 69/9789240013131-eng.pdf Date last accessed: December 2020

2. Sandgren A, Hollo V, Van der Werf MJ. Extra-pulmonary tuberculosis in the European Union and European Economic Area, 2002 to 2011. Euro Surveill 2013; 18 20431.

3. Yang Z, Kong Y, Wilson F, et al. Identification of risk factors for extrapulmonary tuberculosis. Clin Infect Dis 2004; 38: 199-205.

4. Godfrey MS, Friedman LN. Tuberculosis and biologic therapies. Anti-TNF-alpha and beyond. Clin Chest Med 2019; 40: 721-739.

5. Navarra SV, Tang B, Lu L, et al. Risk of tuberculosis with antitumor necrosis factor alpha therapy: substantially higher number of patients in Asia. Int J Rheum Dis 2014; 17: 291-298.
6. Peto HM, Pratt RH, Harrington TA, et al. Epidemiology of extrapulmonary tuberculosis in the United States, 19932006. Clin Infect Dis 2009; 49: 1350-1357.

7. Lewinsohn DM, Leonard MK, LoBue PA, et al. Official American Thoracic Society/Infectious Diseases Society of America / Centers for Disease Control and Prevention Clinical Practice Guidelines: diagnosis of tuberculosis in adults and children. Clin Infect Dis 2017; 64: e1-e33.

8. Tyrell FC, Budnick GE, Elliott T, et al. Probability of negative Mycobacterium Complex cultures based on time to detection of positive cultures: a multicentre evaluation of commercialbroth-based culture systems. J Clin Microbiol 2012; 50 : 3575-3282.

9. Kumari R, Tripathi R, Pandey AP, et al. Rapid screening of MDR-TB in cases of extra pulmonary tuberculosis using Geno Type MTBDTplus. PLoS One 2016; 11: e0159651. 
10. Singh UB, Pandey P, Mehta G, et al. Genotypic, phenotypic and clinical validation of GeneXpert in extra-pulmonary and pulmonary tuberculosis in India. PLoS One 2016; 11: e0149258.

11. Nahid P, Dorman SE, Alipanah N, et al. Official American Thoracic Society / Centers for Disease Control and Prevention / Infectious Disease Society of America Practice Guidelines: Treatment of drug-susceptible tuberculosis. Clin Infect Dis 2016; 63: e147-e195.

12. Touati MM, Darouassi Y, Chihani M, et al. [ENT primitive extranodal tuberculosis: about 15 cases.] Pan Afr Med J 2014; 19: 179-190.

13. Iseri M, Aydýner O, Celik L, et al. Tuberculosis of the parotid gland. J Laryngol Otol 2005; 119: 311-313.

14. Mayosi BM, Wiysonge CS, Ntsekhe $M$, et al. Clinical characteristics and initial management of patients with tuberculous pericarditis in the HIV ara: the Investigation of the Management of Pericarditis in Africa (IMPI Africa) registry. BMC Infect Dis 2006; 6: 2.

15. Chia BL, Choo M, Tan A, et al. Echocardiographic abnormalities in tuberculous pericardial effusion. Am Heart J 1984; 107: 1034-1035.

16. Tuon FF, Litvoc MN, Lopes MIBF. Adenosine desaminase and tuberculous pericarditis: as systemic review with metaanalysis. Acta Trop 2006; 99: 67-74.

17. Mayosi BM, Ntsekhe M, Bosch J, et al. Prednisolone and Mycobacterium indicus pranii in tuberculous pericarditis. N Engl J Med 2014; 371: 1121-1230

18. Sengupta PP, Eleid MF, Khandheiria BK. Constrictive pericarditis. Circ J 2008; 72: 1555-1562.

19. Lewis BS. Real time 2-dimensional echocardiography in constrictive pericarditis. Am J cardiol 1982; 49: 1789-1793.

20. Hurrell DG, Nishimura RA, Higano ST, et al. Value of dynamic respiratory changes in left and right ventricular pressures for the diagnosis of constrictive pericarditis. Circulation 1996; 93 : 2007-2013.

21. Wang ZJ, Reddy GP, Gotway MB, et al. CT and MR imaging of pericardial disease. Radiographics 2003; 23 Spec No: S127-S180.

22. Crump JA, Reller BL. Two decades of disseminated tuberculosis at a university medical center: the expanding role of mycobacterial blood culture. Clin Infect Dis 2003; 37: 1037-1043.

23. Sharma SK, Mohan A. Miliary tuberculosis. Microbiol spectr 2017; 5: 10.1128/microbiolspec.TNMI7-0013-2016.

24. Sharma SK, Mohan A, Sharma A, et al. Miliary tuberculosis: new insights into an old disease. Lancet Infect Dis 2005; 5: 415-430.

25. Shah M, Martinson NA, Chaisson RE, et al. Quantitative analysis of a urine-based assay for detection of lipoarabinomannan in patients with tuberculosis. J Clin Microbiol 2010; 48: 2972-2974.

26. Minion J, Leung E, Talbot E, et al. Diagnosing tuberculosis with urine lipoarabinomannan: systematic review and metaanalysis. Eur Respir J 2011; 38: 1398-1405.

27. Gupta-Wright A, Corbett EL, Van Oosterhout JJ, et al. Rapid urine-based screening for tuberculosis in HIV-positive patients admitted to hospital in Africa (STAMP): a pragmatic multicentre, parallel-group, double-blind, randomised controlled trial. Lancet 2018; 392: 292.

28. Anderson NE, Somaratne J, Mason DF, et al. A review of tuberculous meningitis at Auckland City Hospital. New Zealand J Clin Neurosci 2010; 17: 1018-1022.

29. Mechai F, Bouchaud O. Tuberculous meningitis: challenges in diagnosis and management. Rev Neurol (Paris) 2019; 175: 451-457

30. Sharma P, Garg RK, Verma R, et al. Incidence, predictors and prognostic value of cranial nerve involvement in patients with tuberculous meningitis: A retrospective evaluation. Eur J Intern Med 2011; 22: 289-295.

31. Mogado C, Ruivo N. Imaging meningo-encephalic tuberculosis. EurJ Radiol 2005; 55: 188-192.

32. Thwaites GE, Chau TTH, Stepniewska K, et al. Diagnosis of adult tuberculous meningitis by use of clinical and laboratory features. Lancet 2002; 360: 1287-1292.

33. Tuon FF, Higashino HR, Ferreira Lopes MIB, et al. Adenosine desaminase and tuberculous meningitis. A systematic review with meta-analysis. Scand J Infect Dis 2010; 42: 198-207.

34. Pormohammad A, Nasiri MJ, McHugh TD, et al. A systematic review and meta-analysis of the diagnostic accuracy of nucleic acid amplification tests for tuberculous meningitis. J Clin Microbiol 2019; 57: e01113-e01118.

35. Nhu NTQ, Heemskerk D, Thu DDA, et al. Evaluation of GeneXpert MTB/RIF for diagnosis of tuberculous meningitis. J Clin Microbiol 2014; 52: 226-233.

36. Heemskerk AD, Donovan J, Thu DDA, et al. Improving the microbiological diagnosis of tuberculous meningitis: a prospective, international, multicentre comparison of conventional and modified Ziehl-Neelsen stain, GeneXpert, and culture of cerebrospinal fluid. J Infect 2018; 77: 509-515

37. Prasad K, Singh MB. Corticosteroids for managing tuberculous meningitis. Cochrane Database Syst Rev 2008; 1: CD002244.

38. Twaithes G, Fisher M, Hemingway C, et al. British infection society guidelines for the diagnosis and treatment of tuberculosis of the central nervous system in adults and children. J Infect 2009; 59: 167-187.

39. Donovan J, Figagi A, Imran D, et al. The neurocritical care of tuberculous meningitis. Lancet Neurol 2019; 18: 771-783.

40. Mohanty A, Santosh V, Anandh B, et al. Diagnostic efficacy of stereotactic biopsies in intracranial tuberculomas. Sur Neurol 1999; 52: 252-258.

41. Garg RK, Malhotra HS, Gupta R. Spinal cord involvement in tuberculous meningitis. Spinal Cord 2015; 53: 649-657.

42. Fontanilla JM, Barnes A, Von Reyn CF. Current diagnosis and management of peripheral tuberculous lymphadenitis. Clin Inf Dis 2011; 53: 555-562.

43. Mathiasen VD, Eiset $\mathrm{AH}$, Andersen $\mathrm{PH}$, et al. Epidemiology of tuberculous lymphadenitis in Denmark : a nationwide register-based study. PLoS One 2019; 14: e0221232.

44. Gupta A, Rahman K, Shahid M, et al. Sonographic assessment of cervical lymphadenopathy: role of high-resolution and color doppler imaging. Head Neck 2011; 33: 297-302.

45. Gautam H, Agrawal SK, Verma SK, et al. Cervical tuberculous lymphadenitis: clinical profile and diagnostic modalities. Int J Mycobacteriol 2018; 7: 212-216.

46. Biadglegne F, Tesfaye W, Sack U, et al. Tuberculous lymphadenitis in Northern Ethiopia: in a public health and microbiological perspectives. PloS One 2013; 8: e8198.

47. Salvador F, Los-Arcos I, Sanchez-Montalva A, et al. Epidemiology and diagnosis of tuberculous lymphadenitis in a tuberculosis low-burden country. Medicine (Baltimore) 2015; 94: e509.

48. Mondoni M, Reposi A, Carlucii P, et al. Bronchoscopic techniques in the management of patient with tuberculosis. Int J Infect Dis 2017; 64: 27-37.

49. Chhajed PN, Vaidya PJ, Mandovra NP, et al. EBUS-TBNA in the rapid microbiological diagnosis of drug-resistant mediastinal lymphadenopathy. ERJ Open Res 2019 Nov 15; 5: 00008-2019.

50. Marais S, Roos I, Mitha A, et al. Spinal tuberculosis: clinicoradiological findings in 274 patients. Clin Infect Dis 2018; 67: 89-98.

51. Batirel A, Erdem H, Pehlivanoglu F, et al. The course of spinal tuberculosis (Pott Disease): results of the multinational, multicentre backbone 2 study. Clin Microbiol Infect 2015; 21: 1008.e9-1008.e18.

52. Turgut M. Spinal tuberculosis (Pott's disease): its clinical presentation, surgical management, and outcome. A survey study on 694 patients. Neurosurg Rev 2001; 24: 8-13.

53. Rivas-Garcia A, Sarria-Estrada S, Torrents-Odin C, et al. Imaging findings of Pott's disease. Eur Spine J 2013; 22 Suppl 4: 567-578

54. Currie S, Galea-Soler S, Barron D, et al. MRI characteristics of tuberculous spondylolitis. Clin Radiol 2011; 66: 778-787.

55. Wang G, Dong W, Lan T, et al. Diagnostic accuracy evaluation of the conventional and molecular tests for spinal tuberculosis in a cohort, head-to-head study. Emerg Microbes Infect 2018; 7: 109.

56. Held M, Laubscher M, Zar HJ, et al. GeneXpert polymerase chain reaction for spinal tuberculosis: an accurate and rapid diagnostic tests. Bone Joint J 2014; 96-B: 1366-1369.

\section{Suggested answers}

1. a, c, d.

2. $b$ (in case of miliary tuberculosis with meningitis), $d$.

3. a, c.

4. a, b, c, d. 
57. De Backer Al, Mortele KJ, Vanhoenacker FM, et al. Imaging of extraspinal musculoskeletal tuberculosis. EurJ Radiol 2006 57: $119-130$

58. Sharma A, Pinto B, Dogra S, et al. A case series and review of Poncet's disease, and the utility of current diagnosis criteria. Int J Rheum Dis 2016; 19: 1010-1017.

59. Vaid U, Kane GC. Tuberculous peritonitis. Microbiol Spectr 2017; 5: TNM17-0006-2016.

60. Liu R, Jing L, Tan Y, et al. Multicenter evaluation of the acidfast bacillus smear, mycobacterial culture, Xpert MTB/ RIF assay, and adenosine desaminase for the diagnosis of tuberculous peritonitis in China. Int J Infect Dis 2020; 90 119-124.

61. Guirat A, Affes N, Rejab H, et al. [Role of laparoscopy in the diagnosis of peritoneal tuberculosis in endemic areas.] Med Sante Trop 2015; 25: 87-91.

62. Schinina V, Albarello F, Christofaro M, et al. Diagnostic imaging of hepatic tuberculosis: case series. Int J Tuberc Lung Dis 2018; 22: 779-787.

63. Chog VH, Lim KS. Hepatobiliary tuberculosis. Singapore Med J 2010; 51: 744-751.

64. Bandyopadhyay S, Maity PK. Hepatobiliary tuberculosis. J Assoc Physicians India 2013; 61: 404-407.

65. Hickey AJ, Gounder L, Moosa MY, et al. A systematic review of hepatic tuberculosis with considerations in human immunodeficiency virus co-infection. BMC Infect Dis 2015; 15: 209-220.

66. Alvares JF, Devarbhavi H, Makhija P, et al. Clinical, colonoscopic, and histological profile of colonic tuberculosis in a tertiary hospital. Endoscopy 2005; 37: 351-356.

67. Kirsch R, Pentecost M, de M Hall P, et al. Role of colonoscopic biopsy in distinguishing between Crohn's disease and intestinal tuberculosis. J Clin Pathol 2006; 59: 840-844.

68. Kulchavenya $E$. Best practice in the diagnosis and management of urogenital tuberculosis. Ther Adv Urol 2013; 5: 143-151.

69. Gaudiano C, Tadolini M, Busato F, et al. Multidetector CT urography in urogenital tuberculosis: use of reformatted images for the assessment of the radiological findings. A pictorial essay. Abdom Radiol (NY) 2017; 42: 2314-2324.

70. Thangamani M, Matcha J, Edwin MF, et al. Acute renal failure due to rifampicin: a study of 25 patients. Am J Kidney Dis 2002; 40: 690-696.

71. Guo YK, Yang ZG, Li Y, et al. Addison's disease due to adrenal tuberculosis : contrast-enhanced $\mathrm{CT}$ features and clinical duration correlation. EurJ Radiol 2007; 62: 126-131.

72. Zhang XC, Yang ZG, Min PQ, et al. Addison's disease due to adrenal tuberculosis: MRI features. Abdom Imag 2008; 33 : 689-694.

73. Chen Q, Chen WC, Hao F. Cutaneous tuberculosis: a great imitator. Clin Dermatol 2019; 37: 192-199. 